corresponding to the LCI values given), and VV\% $=89.2(2.6)$. 2 of the 4 CF patients had normal sitting and supine LCI and 2 of 3 had no evidence of gas trapping, but ${ }^{3} \mathrm{He}$ MRI showed clear focal ventilation defects in all patients (b-d).

Conclusions High quality ${ }^{3} \mathrm{He}$ MRI images can be produced in young children. Preliminary results suggest that hyperpolarised ${ }^{3} \mathrm{He}$ ventilation MRI may be more sensitive to early ventilation changes in CF than LCI or conventional lung function tests.

Acknowledgements CF Trust funding

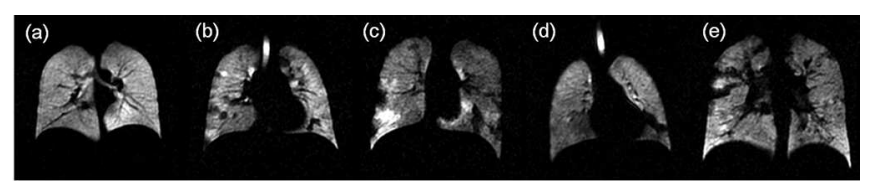

Abstract S8 Figure 1. Ventilation images from (a) a healthy volunteer and (b-e) patients with mild CF.

\section{S9 SIFT-MS ANALYSIS AS A NON-INVASIVE DETERMINANT OF PSEUDOMONAS AERUGINOSA INFECTION IN PATIENTS WITH CYSTIC FIBROSIS}

${ }^{1} \mathrm{R}$ Pabary, ${ }^{2} \mathrm{~S}$ Kumar, ${ }^{2} \mathrm{~J}$ Huang, ${ }^{3}$ EWFW Alton, ${ }^{1} \mathrm{~A}$ Bush, ${ }^{2} \mathrm{~GB}$ Hanna, ${ }^{3} \mathrm{JC}$ Davies; Royal Brompton and Harefield Hospital Foundation Trust, London, United Kingdom?; Department of Cancer and Surgery, Imperial College, London, United Kingdom²; National Heart and Lung Institute, Imperial College, London, United Kingdom ${ }^{3}$

\subsection{6/thoraxjnl-2013-204457.15}

Background There is evidence that Pseudomonas aeruginosa $(\mathrm{Pa})$ produces volatile organic compounds (VOCs) such as hydrogen cyanide $(\mathrm{HCN})$ and 2-aminoacetophenone (2-AA). VOCs in exhaled breath are therefore proposed as potential biomarkers of infection. We hypothesised that selective ion-flow mass spectrometry (SIFT-MS) breath analysis might allow discrimination of $\mathrm{CF}$ patients with $(\mathrm{CF}+\mathrm{Pa})$ and without $\mathrm{Pa}(\mathrm{CF}-\mathrm{Pa})$.

Methods 79 adults $(31 \mathrm{CF}+\mathrm{Pa}, 22 \mathrm{CF}-\mathrm{Pa}$ and 26 healthy controls) provided starved, single tidal exhalation breath samples into Nalophan ${ }^{\mathrm{TM}}$ bags. Quantification of 15 VOCs was performed within two hours on SIFT-MS. All results are presented as (median parts-per-billion by volume [IQR]).

Results 2-AA was significantly higher in $\mathrm{CF}+\mathrm{Pa}$ than $\mathrm{CF}-\mathrm{Pa}$ (5.0 [3.4-7.1] vs. $1.3[0.0-3.2], \mathrm{p}<0.01)$. However, there was significant overlap and median co-efficient of variation was $35.41 \%$; clinical utility is therefore questionable.

Dimethyl disulphide was also significantly higher in $\mathrm{CF}+\mathrm{Pa}$ (95.2 [41.3-211.2 vs. 35.5 [22.1-79.8], p < 0.01). When combined with 2-AA, area under ROC curve was 0.867 .

Counter to our sputum results, there was no difference in $\mathrm{HCN}$ between $\mathrm{CF}+\mathrm{Pa}$ and CF-Pa (8.1 [5.0-11.9] vs. 6.9 [4.411.0], $\mathrm{n} / \mathrm{s})$ or between all CF patients and healthy controls $(7.8$ [4.9-11.5] vs. 7.0 [4.6-11.5], $\mathrm{n} / \mathrm{s})$.

Our early in vitro data showed decreased butanol above $\mathrm{Pa}$ cultures, suggesting consumption. This was replicated in breath with lower levels in CF + Pa vs. CF-Pa (37.4 [24.3-87.6] vs. 91.7 [46.9-143.7], $\mathrm{p}<0.05$ ).

Of VOCs likely to be of host origin, isoprene was increased in CF vs. controls (108.0 [83.4-195.5] vs. 69.6 [46.9-89], p < $0.01)$ with no difference between $\mathrm{CF}+\mathrm{Pa}$ vs. CF-Pa. Acetone was reduced in CF (269.9 [161.9-356.4] vs. 324.9 [236.7598.9], $\mathrm{p}<0.01$ ).

Conclusions 2-AA is a potential biomarker of $\mathrm{Pa}$ infection but clinical applicability is uncertain. Dimethyl disulphide and butanol also show promise. Mouth-exhaled HCN assessed by SIFT-
MS does not appear to fulfil its promise as a Pa biomarker. Other VOCs assessed were either similar between Pa groups or different between healthy controls and CF, but unable to differentiate between $\mathrm{Pa}$ status. This study provides proof-of-concept for the development of a non-invasive tool with which to screen for lower airway bacterial infection in CF though a clinically applicable test remains some way off.

\section{S10 LUNG CLEARANCE INDEX (LCI) AND PSEUDOMONOUS AERUGINOSA IN ADULTS AND CHILDREN WITH CYSTIC FIBROSIS (CF)}

${ }^{1} \mathrm{~K}$ O'Neill, ${ }^{2} \mathrm{~J}$ M Bradley, ${ }^{3} \mathrm{I}$ Bradbury, ${ }^{1} \mathrm{E}$ Johnston, ${ }^{4} \mathrm{~A}$ Reid, ${ }^{4} \mathrm{~J}$ McCaughan, ${ }^{4} \mathrm{JE}$ Moore, ${ }^{1} \mathrm{MM}$ Tunney, ${ }^{1} \mathrm{JS}$ Elborn; 'Queen's University Belfast, CF \& Airways Microbiology Research Group, Belfast, United Kingdom; ${ }^{2}$ Centre for Health and Rehabilitation Technologies, University of UIster., Belfast, United Kingdom; ${ }^{3}$ Frontier Science Ltd, Scotland, United Kingdom; ${ }^{4}$ Belfast Health and Social Care Trust, Belfast, United Kingdom

\subsection{6/thoraxjnl-2013-204457.16}

Introduction LCI obtained from multiple breath washout (MBW) is a sensitive measure of ventilation inhomogeneity in CF. Persistent colonisation with $P$. aeruginosa is associated with a decline in LCI in children (Kraemer et al. 2006). Further research is required to investigate the relationship between airways infection and LCI in adults.

Objective To investigate the sensitivity of LCI to $P$. aeruginosa in adults and children compared with $\mathrm{FEV}_{1} \%$ pred and $\mathrm{FEF}_{25-75} \%$ pred.

Methods Stable CF patients from adult \& paediatric Northern Ireland CF centres were recruited. LCI was derived from MBW, using $0.2 \% \mathrm{SF}_{6}$ and a modified Innocor ${ }^{\mathrm{TM}}$ device. P. aeruginosa status was determined from routine diagnostic culture of a sputum sample or deep throat swab. Patients categorised as having $P$. aeruginosa infection met the criteria of chronic infection as defined by the Leeds criteria (Lee et al. 2003).

Analysis Receiver-operator characteristic (ROC) curves and area under the receiver operating curves $\left(\mathrm{AUC}_{\mathrm{ROC}}\right)$ indicate the level of sensitivity and specificity where $1.0=$ perfect discrimination considering sensitivity and specificity.

Results Sixty-seven adults were recruited (39M), median (IQR) age 27 (16) years. Mean (SD) $\mathrm{FEV}_{1} \%$ pred 71.8 (20.3), median (IQR) $\mathrm{FEF}_{25-75} \%$ pred 40.0 (46.7) and mean (SD) LCI 10.3 (3.0) lung volume turnovers. $49 \%$ had $P$. aeruginosa infection.

Forty-three children were recruited (24M), mean (SD) age 11.7 (3.4) years. Mean (SD) $\mathrm{FEV}_{1} \%$ pred 85.2 (16.6), mean (SD) $\mathrm{FEF}_{25-75} \%$ pred 66.0 (27.6) and mean (SD) LCI was 7.8 (1.8) lung volume turnovers. $16 \%$ had $P$. aeruginosa infection

Compared to $\mathrm{FEV}_{1} \%$ pred and $\mathrm{FEF}_{25-75} \%$ pred, LCI had the greatest sensitivity and specificity to discriminate between $\mathrm{CF}$ patients with and without $P$. aeruginosa in both adults and children. Adult $\mathrm{AUC}_{\mathrm{ROC}}(\mathrm{SE})$ for LCI $=0.82(0.05), \mathrm{p}<0.0001$, compared with $\mathrm{FEV}_{1} \%$ pred $=0.66(0.07), \mathrm{p}=0.021$ and $\mathrm{FEF}_{25-75} \%$ pred $=0.64(0.07), \mathrm{p}=0.044$ (Figure 1). Child $\mathrm{AUC}_{\mathrm{ROC}}(\mathrm{SE})$ for LCI $=0.85(0.10), \mathrm{p}=0.004$, compared with $\mathrm{FEV}_{1}$ \%pred $=0.80(0.12), \mathrm{p}=0.014$ and $\mathrm{FEF}_{25-75} \%$ pred $=0.67(0.13), \mathrm{p}=0.152$.

Conclusion LCI is more sensitive and specific to the presence of $P$. aeruginosa airways infection across the age groups in CF compared with spirometry.

This project was funded by a US-Ireland Project Partnership Grant. 


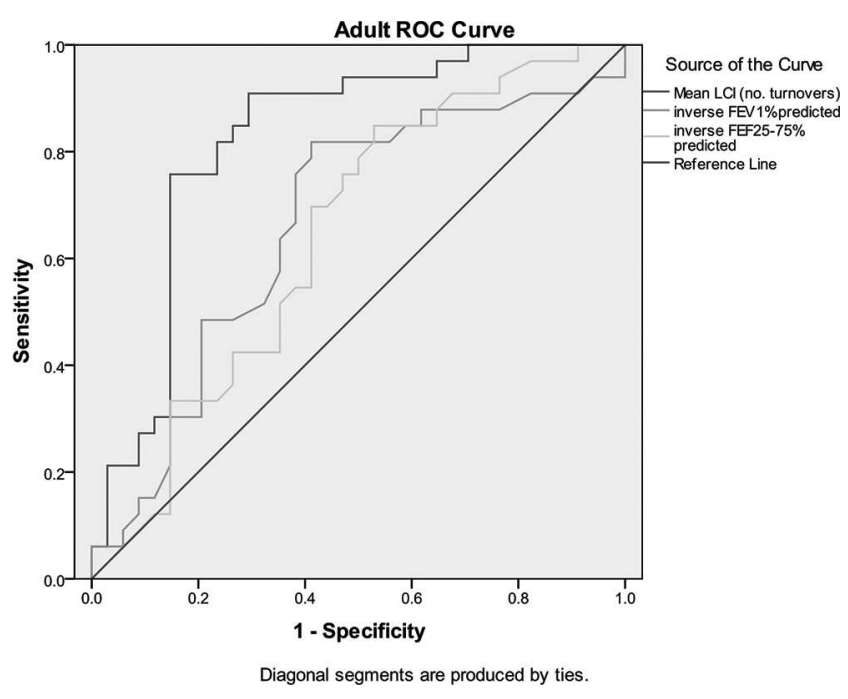

Abstract S10 Figure 1. Adult ROC curve

\section{REFERENCES}

Kraemer, $R$ et al 2006, Respiratory

Research. Lee, T W R et al 2003, Journal of Cystic Fibrosis.

\section{S11 FEASIBILITY OF CONDUCTING COMPLEX PHYSIOLOGICAL MEASUREMENTS IN LONDON PRIMARY SCHOOLS: THE SIZE \& LUNG FUNCTION IN CHILDREN (SLIC) STUDY}

${ }^{1} S$ Lum, ${ }^{1} S$ Sonnappa, ${ }^{2} \mathrm{~A}$ Wade, ${ }^{1} \mathrm{~J}$ Kirkby, ${ }^{1} \mathrm{R}$ Bonner, ${ }^{1} \mathrm{~S}$ Lee, ${ }^{1} \mathrm{~V}$ Bountziouka, ${ }^{1} \mathrm{~S}$ Legg, ${ }^{1} \mathrm{E}$ Raywood, 'D Sears, ${ }^{1} \mathrm{P}$ Cottam, ${ }^{1} \mathrm{~J}$ Stocks; ${ }^{1}$ Portex Respiratory Unit, UCL Institute of Child Health, London, UK; ${ }^{2}$ Centre for Paediatric Epidemiology and Biostatistics, UCL Institute of Child Health, London, UK

\subsection{6/thoraxjnl-2013-204457.17}

Despite recognised ethnic differences in lung function, most reference ranges are based on White subjects. Ethnic minorities comprise $40 \%$ of the London population, which impacts on healthcare provision. Even when available, selection of appropriate equations is complicated by the increase in admixed populations and complexities of defining 'ethnicity'. As part of the Wellcome Trust SLIC study (www.ucl.ac.uk/slic) to determine the extent to which body shape, size and composition contributes to ethnic differences in lung function, we examined the feasibility of conducting complex physiological measurements in a multiethnic population of London primary school children.

Methods 14 London schools participated in the study. Science workshops were presented one week prior to commencing assessments. Consent forms and information packs were distributed to all children. All children with parental consent were eligible and were categorised into 4 broad ethnic groups: White; Black; South-Asian (Indian subcontinent) and Other/mixed. Assessments were performed at school in 5-11 year-old children and included detailed anthropometry, 3D phototonic scan for body shape; body composition; spirometry and saliva samples (cotinine and DNA analysis).

Results Parental consent for anthropometry and spirometry was obtained in 54\% of those approached. Amongst these, $88 \%$ and $96 \%$ provided specific consent for DNA samples and access to GP records respectively (Table 1$)$. Assessments were performed in 2175 children (mean (SD)age: 8.22(1.63); 34\%White; 29\% Black; 25\%South-Asian; 12\%Other/mixed ethnicities), 1045 $(48 \%)$ of whom had follow-up assessments a year later. Preliminary analysis indicates: $18 \%$ had chronic respiratory illness or
Abstract S11 Table 1. Consent, asthma status \&spirometry success rates of study population

\begin{tabular}{lllll}
\hline & White & Black & S-Asian & Other/mixed \\
\hline Total Tested (\% boys) & $742(49.7 \%)$ & $629(43.6 \%)$ & $540(48.7 \%)$ & $264(46.6 \%)$ \\
DNA consent & $89.3 \%$ & $84.0 \%$ & $85.8 \%$ & $92.2 \%$ \\
GP record access consent & $97.1 \%$ & $93.8 \%$ & $93.6 \%$ & $97.4 \%$ \\
Asthma: ever & $11.6 \%$ & $11.1 \%$ & $8.9 \%$ & $19.7 \%$ \\
Asthma: current & $5.5 \%$ & $4.6 \%$ & $5.6 \%$ & $7.2 \%$ \\
Totalspirometry $^{\text {a }}$ & $533(71.8 \%)$ & $435(69.2 \%)$ & $411(76.1 \%)$ & $195(73.9 \%)$ \\
\hline
\end{tabular}

Data presented as \%.

Abbreviations: DNA: Deoxyribonucleic acid (for genetic ancestry); GP: General Practitioner; Current asthma: defined as those having symptoms and/or asthma medication over the past 12 months;

abased on data from healthy children and after exclusions from poor health and poor performance.

acute symptoms at time of test. $12 \%$ children had a diagnosis of 'asthma ever', with 6\% having current asthma (Table 1). Acceptable spirometry ${ }^{1}$ was obtained from 1574(72\%) healthy children.

Summary Conducting a field study to undertake complex physiological measurements is feasible even in young children. However, the relatively high prevalence of chronic or acute respiratory disease at time of testing in this age group, combined with exclusions due to technically unsatisfactory spirometry means that results from $\sim 30 \%$ of children may be excluded if analysis of results is to be based on a 'healthy' population. Such factors must be accounted for when designing respiratory field studies to ensure adequate sample size to reach definitive conclusions.

\section{REFERENCE}

1. Kirkby et al. Pediatr Pulmonol 2008.

\section{Interstitial lung disease: clinical}

\section{S12 INTERSTITIAL LUNG DISEASE MULTIDISCIPLINARY DISCUSSION: SIX YEARS OF DATA FROM A TERTIARY SERVICE}

A Wright, J Helm, L Spencer, C Leonard, P Bishop, M Greaves, N Chaudhuri; University Hospital of South Manchester NHS Trust, Manchester, England

\subsection{6/thoraxjnl-2013-204457.18}

Introduction Accurate diagnosis in Interstitial Lung Disease (ILD) is vital in optimising patient management. An integrated approach involving a multidisciplinary team (MDT) of physicians, radiologists and pathologists is strongly advised in ATS/ ERS guidelines. ${ }^{1}$ This has been shown to improve diagnostic confidence. ${ }^{2}$ Consensus diagnosis post multidisciplinary team discussion often differs from that reached by individual clinicians. Our centre, which provides a tertiary interstitial lung disease service in the North of England, implemented multidisciplinary discussion in 2005. Our patient cohort is larger than series previously presented at both national and international respiratory meetings. Literature search also did not identify any published data with either an equal or greater patient population.

Aims To review interstitial lung disease MDT outcomes and to determine if discussion resulted in a change of diagnosis and whether this impacted on subsequent patient management.

Methods Retrospective review of both patient clinical notes and MDT outcomes from 2005 to 2013 was performed. Data from 